RHIC/AP/158

August, 1998

\title{
RHIC head-tail stability with resistive wall wake fields
}

\author{
C. Lee, S. Peggs
}

\section{Introduction}

Consider two highly relativistic particles, with charges $q_{1}$ and $q_{2}$, that circulate RHIC with horizontal and longitudinal displacements of $\left(x_{1}, z_{1}\right)$ and $\left(x_{2}, z_{2}\right)$ from the center of the same bunch. If they pass through a short device (at azimuthal location $s=0$ ) that generates a wake field, the equation of motion of particle 2 is written as

$$
x_{2}^{\prime \prime}+K(s) x_{2}=\frac{q_{1} q_{2}}{m c^{2} \gamma} V_{1}\left(z_{1}-z_{2}\right) x_{1} \delta(s)
$$

where a prime denotes differentiation with respect to azimuthal coordinate $s$, $K(s)$ is the quadrupole focusing, $m$ is the particle mass, and $c$ and $\gamma$ are the usual relativistic quantities. The coupling between the particles, integrated across the device, is given by $V_{1}\left(z_{1}-z_{2}\right)$, the "transverse wake potential". Causality demands that the leading particle drives the trailing particle, but not vice versa, so that $V_{1}=0$ if $z_{1}<z_{2}$ (and if multi-turn wakes are neglected). Also, the wake potential is always positive for particles that are very close together, $V_{1}(+\epsilon)>0$, so that a trailing particle that is in phase with the source particle is defocused.

In general there are $10^{9}$ or more particles per bunch in RHIC. The numerical

code KRAKEN models their motion by giving each of $N_{m} \lesssim 200$ macro-particles an angular kick once per turn [1]. If there are $N_{b}$ ions of atomic number $Z$ and atomic weight $A$ in each bunch, then the net angular kick given to particle $i$ is

$$
\Delta x_{i}^{\prime}=\frac{N_{b} Z^{2} e^{2}}{A m_{u} c^{2} \gamma} \frac{1}{N_{m}} \sum_{j=1}^{N_{m}} V_{1}\left(z_{j}-z_{i}\right) x_{j}
$$

Longitudinal motion in KRAKEN is inexorable and linear, so that

$$
z_{i}=\widehat{z_{i}} \sin \left(\frac{2 \pi t}{T_{s}}+\phi_{s}\right)
$$

where $\widehat{z_{i}}$ is the longitudinal amplitude, $t$ is the time in turns, $T_{s}$ is the synchrotron period, and $\phi_{s}$ is the initial phase at $t=0$. 


\subsection{Resistive wall wake}

Even a smooth conducting beam pipe generates a wake field - the "resistive wall" wake field. The transverse resistive wall wake potential due to a circular beam pipe of length $L$ is given by

$$
\begin{array}{lll}
V_{1}(z)=\frac{8 L}{r^{4} \epsilon_{0}(4 \pi)^{3 / 2}} z & 0<z \ll z_{c} \\
V_{1}(z)=\frac{2 L}{\pi r^{3}} \sqrt{\frac{c}{4 \pi \epsilon_{0} \sigma}} z^{-1 / 2} & & z \gg z_{c}
\end{array}
$$

where $r$ and $\sigma$ are the radius and conductivity of the pipe [2]. The critical length $z_{c}$, given by

$$
z_{c}=\left(\frac{c \epsilon_{0}}{\sigma}\right)^{1 / 3} r^{2 / 3}
$$

tends to be very short. For example, $z_{c}=0.12 \mathrm{~mm}$ for the dominant (cold) beam pipe in RHIC, with $L=2955 \mathrm{~m}, r=0.0346 \mathrm{~m}$, and $\sigma=2.0 \Omega^{-1} \mathrm{~m}^{-1}$.

It turns out that RHIC is most vulnerable to head-tail effects with proton bunches at injection, with nominal parameters given in Table 1. These parameters are used as defaults in the results presented below.

Table 1: RHIC parameters during proton injection.

\begin{tabular}{lcc}
\hline Parameter & units & value \\
& & \\
\hline & & $1.0 \times 10^{11}$ \\
Bunch population, $N_{b}$ & & 31.17 \\
Lorentz factor, $\gamma$ & & 22.89 \\
Transition gamma, $\gamma_{T}$ & $\mathrm{~m}$ & 30.0 \\
Average device beta, $\beta_{D}$ & $\mathrm{~m}$ & 3833.8 \\
Circumference, $C$ & turns & 1414 \\
Synchrotron period, $T_{s}$ & & $4.66 \times 10^{-3}$ \\
RMS momentum error, $\sigma_{p} / p$ & $\mathrm{~m}$ & 0.353 \\
RMS bunch length, $\sigma_{z}$ & & \\
& & \\
\hline
\end{tabular}

A numerical model with one kick per turn is still valid even if the device is long - for example, a continuous beam pipe - if the synchrotron period measured in turns is long $\left(T_{s} \gg 1\right)$, and if the beta function at the kick point is the average beta over the device. 


\section{Two macro-particles}

In the simplest case there are only two macro-particles, with longitudinal oscillations of the same amplitude $\widehat{z}$ that are out of phase, so that

$$
z_{1}=-z_{2}=\widehat{z} \sin \left(\frac{2 \pi t}{T_{s}}+\phi_{s}\right)
$$

With linearized longitudinal motion, the longitudinal amplitude $\widehat{z}$ is related to the relative momentum amplitude $\widehat{\delta}$ through the relationship

$$
\beta_{z} \equiv \frac{\widehat{z}}{\widehat{\delta}}=\frac{\eta C T_{s}}{2 \pi}
$$

where $C$ is the circumference of the accelerator and the slip factor $\eta$ is given by

$$
\eta=\left|\frac{1}{\gamma_{T}^{2}}-\frac{1}{\gamma^{2}}\right|
$$

in which $\gamma_{T}$ is the transition gamma. Horizontal motion is concisely described using phasor notation to represent the normalized coordinates

$$
\tilde{x} \equiv x+i x^{\prime}
$$

since then, in the absence of wake fields, the motion from turn $t$ to $t+1$ is just

$$
\tilde{x}(t+1)=R \tilde{x}(t)
$$

where $R$ represents the normalized phase space rotation

$$
R=\exp \left(i 2 \pi Q_{x}\right)
$$

and $Q_{x}$ is the horizontal betatron tune.

The motion of the two macro-particles through one synchrotron period, including wake fields, is analytically predicted to be $[2,3,4]$

$$
\left(\begin{array}{c}
\tilde{x_{1}} \\
\tilde{x_{2}}
\end{array}\right)_{t+T_{S}}=\left(\begin{array}{cc}
R^{T_{S}} & 0 \\
0 & R^{T_{S}}
\end{array}\right)\left(\begin{array}{cc}
1-\Upsilon^{2} & i \Upsilon \\
i \Upsilon & 1
\end{array}\right)\left(\begin{array}{c}
\tilde{x_{1}} \\
\tilde{x_{2}}
\end{array}\right)_{t}
$$

It is remarkable that the analytic model is completely described, for an arbitrary wake potential, by two parameters - the betatron phase advance per synchrotron period, $2 \pi Q_{x} T_{s}$, and the dimensionless complex "head-tail parameter", $\Upsilon$,

$$
\begin{aligned}
& \Upsilon(\chi, \widehat{z})=\Upsilon_{R}+i \Upsilon_{I}= \\
& \quad \frac{\beta_{D} N_{b} Z^{2} e^{2}}{4 A m_{u} c^{2} \gamma} \int_{t=0}^{T_{s} / 2} V_{1}\left(z_{1}-z_{2}\right) \exp \left[i\left(2 \chi \widehat{\delta} T_{s}\right) \sin \left(\frac{2 \pi t}{T_{s}}\right)\right] d t
\end{aligned}
$$


where $\beta_{D}$ is the Twiss function at the device, $t$ is time in turns, and $\chi=d Q / d \delta$ is the chromaticity [2]. Note that the second matrix in Eqn. 13 appears to treat particles 1 and 2 unequally, since the two matrix elements on the diagonal are different. This is an artifact of choosing $\phi_{s}=0$, with particle 1 about to take the lead from particle 2 at time $t=0$.

When the values of Table 1 are substituted into Eqn. 14, with $\widehat{\delta}=\sigma_{p} / p$ and $\widehat{z}=\sigma_{z}$, they lead to the variation of $\Upsilon$ with chromaticity recorded in Figure 1 . The maximum value of $\Upsilon_{R}=0.3003$. This implies that nominal proton bunches at injection are about an order of magnitude short of strong head-tail instability, which occurs when $\Upsilon_{R}=2$ (see below).

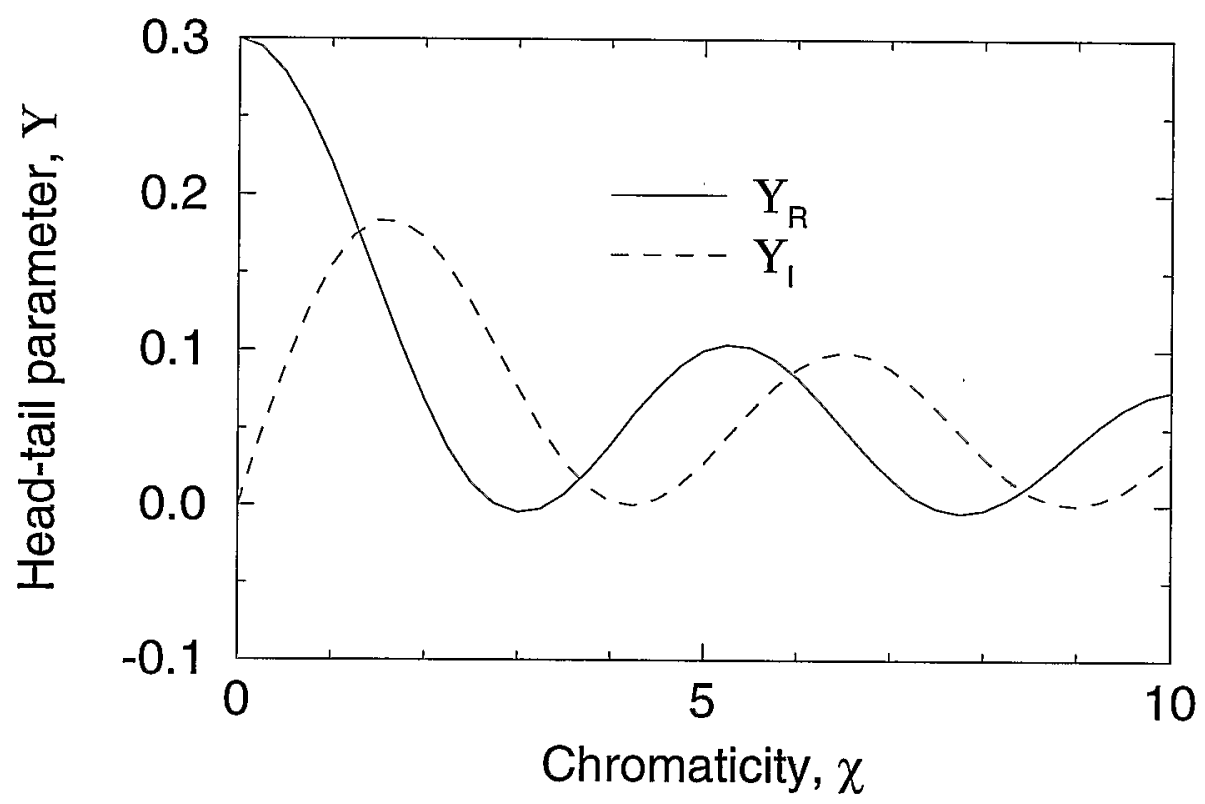

Figure 1: Head-tail parameter $\Upsilon$ versus chromaticity $\chi$ for protons at injection, due to the transverse resistive wall wake.

Various authors have reported analytical (and simulation) head-tail results for the particular case of a step function wake potential[2, 3, 4, 5].

$$
\begin{aligned}
& V_{1}(z)=V_{1} \quad z>0 \\
& V_{1}(z)=0 \quad z<0
\end{aligned}
$$

In this case it is easy to explicitly evaluate Eqn 14 . The analytical results reported by these authors (parameterized by $\Upsilon$ ) are readily adapted to a general wake field, since it is straightforward to evaluate Eqn. 14 numerically.

It is less elegant but more explicit to rewrite Eqn. 13 in 4-dimensional real 
space, so that

$$
\begin{aligned}
& \left(\begin{array}{l}
x_{1} \\
x_{1}^{\prime} \\
x_{2} \\
x_{2}^{\prime}
\end{array}\right)_{t+T_{s}}=\left(\begin{array}{rrrr}
c & s & 0 & 0 \\
-s & c & 0 & 0 \\
0 & 0 & c & s \\
0 & 0 & -s & c
\end{array}\right) \times \\
& \left(\begin{array}{cccc}
1-\Upsilon_{R}^{2}+\Upsilon_{I}^{2} & 2 \Upsilon_{R} \Upsilon_{I} & -\Upsilon_{I} & -\Upsilon_{R} \\
-2 \Upsilon_{R} \Upsilon_{I} & 1-\Upsilon_{R}^{2}+\Upsilon_{I}^{2} & \Upsilon_{R} & -\Upsilon_{I} \\
-\Upsilon_{I} & -\Upsilon_{R} & 1 & 0 \\
\Upsilon_{R} & -\Upsilon_{I} & 0 & 1
\end{array}\right)\left(\begin{array}{c}
x_{1} \\
x_{1}^{\prime} \\
x_{2} \\
x_{2}^{\prime}
\end{array}\right)_{t}
\end{aligned}
$$

where $c=\cos \left(2 \pi Q_{x} T_{s}\right)$ and $s=\sin \left(2 \pi Q_{x} T_{s}\right)$. Since the horizontal motion is exactly linear - both in the analytical model and in the numerical model - the two macro-particle problem now reduces simply to finding the eigenvalues and the eigenmodes of Eqn. 17.

\subsection{Eigenmotion in the analytical model}

If the chromaticity $\chi$ is zero, the motion of two macro-particles is stable for increasing $N_{b}$ until $\operatorname{Re} \Upsilon(0, \widehat{z})=2$, when the strong head-tail threshold is passed. Unstable motion above this threshold' has a rise time of $\tau \sim T_{s}$, the time scale on which the macro-particles exchange their "drive" and "response" roles. The strong head-tail instability, also known as the "transverse mode coupling", "transverse turbulent", or "transverse microwave" instability, has only been observed at electron storage rings.

When a non-zero chromaticity $\chi$ is introduced, one of the real eigenmodes grows and the other is damped with a slow time scale $\tau \gg T_{s}$, even for modest values of $\Upsilon$. This is the head-tail instability. The eigenmode growth rates, per turn, are

$$
\tau_{ \pm}^{-1}=\mp \frac{\operatorname{Im} \Upsilon(\chi, \widehat{z})}{T_{s}}
$$

These results hold for a general transverse wake field. Note that $\tau_{+}^{-1}$ is made negative and the " + " mode is stabilized (above transition) by a slightly positive chromaticity.

The "+" mode has the two macro-particles oscillating transversely in phase with each other, while the "-" mode has them out of phase. The analysis presented above assumes that $\phi_{s}$, the initial longitudinal phase (in Eqn. 7), is zero. If $\phi_{s}$ is nonzero, the transformation in one synchrotron period will be different from Eqn. 17, so that the eigenmodes will change while the eigenvalues remain unchanged. Thus, the two natural physical observables - the amplitude of the center of charge and the RMS beam size - do not represent the pure eigenmodes in the general case. 


\subsection{Eigenmotion in KRAKEN}

For two macro-particles, the simulation results generated by KRAKEN are quite close to those predicted in the analytical model. For example, when we consider the case when $\phi_{s}=0$ in Eqn. 7, and all the parameters are the same as in Table 1, the transformation matrix in one synchrotron period for zero chromaticity is found by KRAKEN to be

$$
\left(\begin{array}{l}
x_{1} \\
x_{1}^{\prime} \\
x_{2} \\
x_{2}^{\prime}
\end{array}\right)_{t+T_{S}}=\left(\begin{array}{rrrr}
-.771406 & -.487551 & -.160869 & .247120 \\
.491563 & -.771406 & -.249103 & -.154043 \\
-.154043 & .247120 & -.844328 & -.535827 \\
-.249103 & -.160869 & .535827 & -.844328
\end{array}\right)\left(\begin{array}{l}
x_{1} \\
x_{1}^{\prime} \\
x_{2} \\
x_{2}^{\prime}
\end{array}\right)_{t}
$$

while the transformation of Eqn. 17 with $\Upsilon=0.3003$ is

$$
\left(\begin{array}{l}
x_{1} \\
x_{1}^{\prime} \\
x_{2} \\
x_{2}^{\prime}
\end{array}\right)_{t+T_{S}}=\left(\begin{array}{rrrr}
-.768338 & -.487603 & -.160748 & .253298 \\
.487603 & -.768338 & -.253298 & -.160748 \\
-.160748 & .253298 & -.844328 & -.535827 \\
-.253298 & -.160748 & .535827 & -.844328
\end{array}\right)\left(\begin{array}{l}
x_{1} \\
x_{1}^{\prime} \\
x_{2} \\
x_{2}^{\prime}
\end{array}\right)_{t}
$$

For other cases with nonzero chromaticities, the KRAKEN simulation is also in good agreement with the analytical model. The slight difference between the matrices results from the fact that KRAKEN uses discrete integration from turn to turn, while the analytical model uses a continuous integration.

Fig. 2 and Fig. 3 show the variation of beam amplitude and RMS beam size with time in units of synchrotron periods for the case of zero initial phase $\left(\phi_{s}=0\right)$ in Eqn. 7 with different chromaticities. Although these two physical observables represent the eigenmodes in an approximate way in the analytical model, the amplitude (an approximation of the " + " eigenmode) is not stabilized as well for larger synchrotron periods since it is not a pure eigenmode. For the RMS beam size, the effect of its small component of "+" mode is negligible because the mode with strongest positive eigenvalue will dominate in the long run. Other initial phases $\phi_{s}$ of the longitudinal oscillation term also lead to the same behavior, eventually.

\section{Many macro-particles}

Our two macro-particle model in KRAKEN is easily generalized to many macroparticles by the same algorithm. Again, we assume that there are $N_{m}$ macroparticles in each beam and there is no coupling between horizontal and vertical oscillations. The macro-particles have longitudinal oscillations with phases uniformly distributed over $2 \pi$. That is, we have

$$
z_{n}=\widehat{z} \sin \left(\frac{2 \pi t}{T_{s}}+\frac{2 \pi(n-1)}{N_{m}}+\phi_{s}\right)
$$




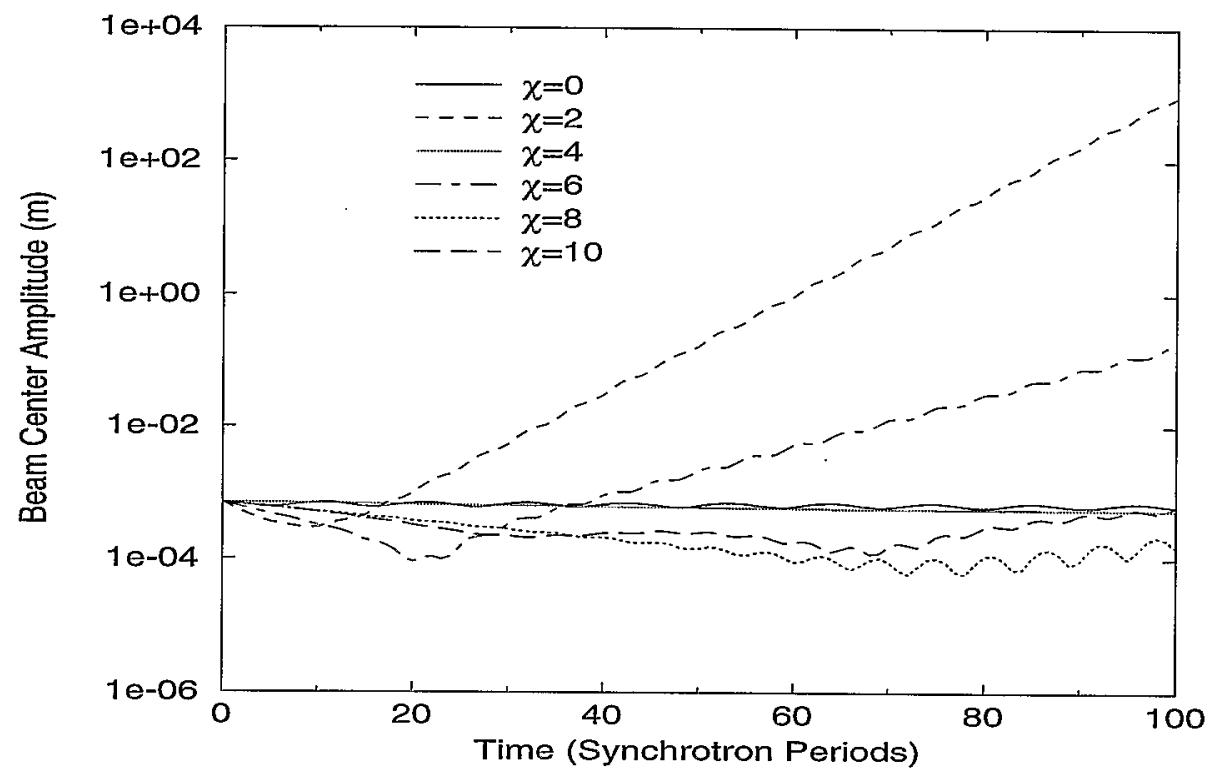

Figure 2: Beam amplitude versus time with $N_{m}=2$ macro-particles and the parameters of Table 1.

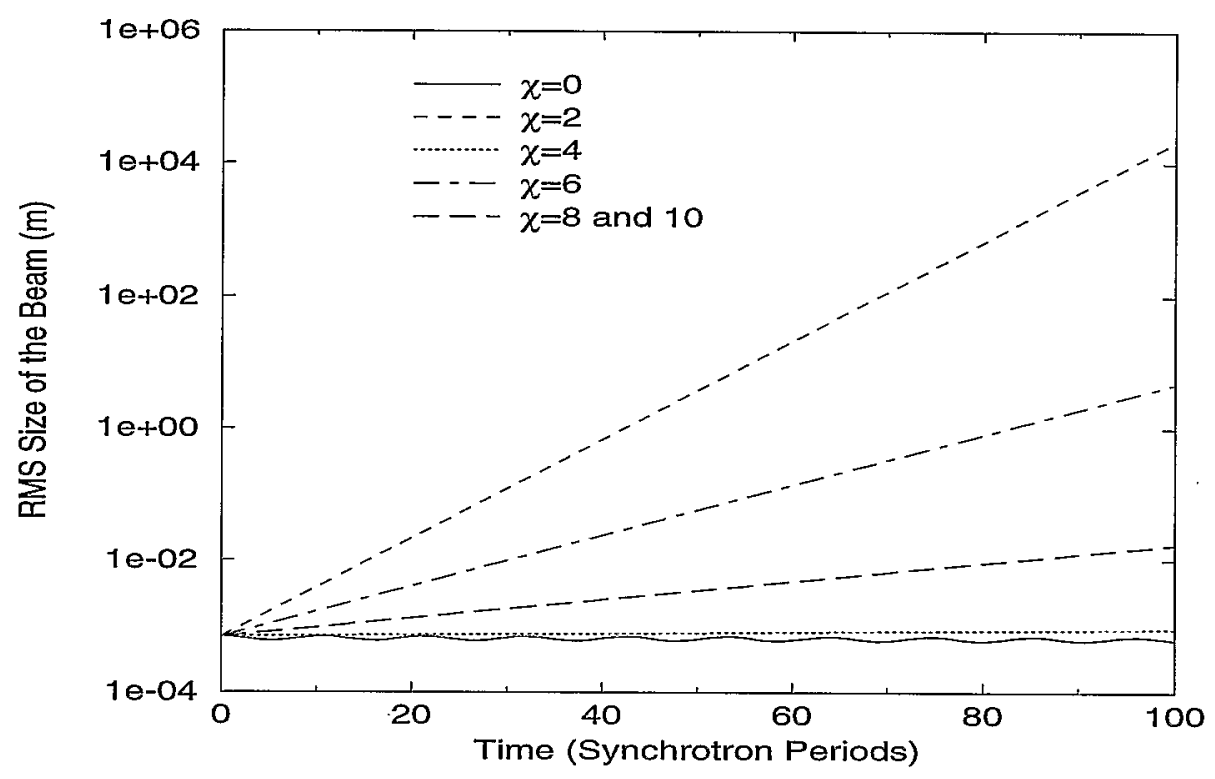

Figure 3: RMS beam size versus time with $N_{M}=2$ macro-particles and the parameters of Table 1. 
for $n=1,2, \ldots, N_{m}-1, N_{m}$. Since the speed of the macro-particles is close to $c$, we can assume that a macro-particle is influenced only by those macroparticles in front of it. For a large number of macro-particles with longitudinal oscillations given by Eqn. 21, $\phi_{s}$ has little effects on the eigenmodes. We believe that in the real beam the transverse eigenmodes would also be independent of the details of the initial phase distribution.

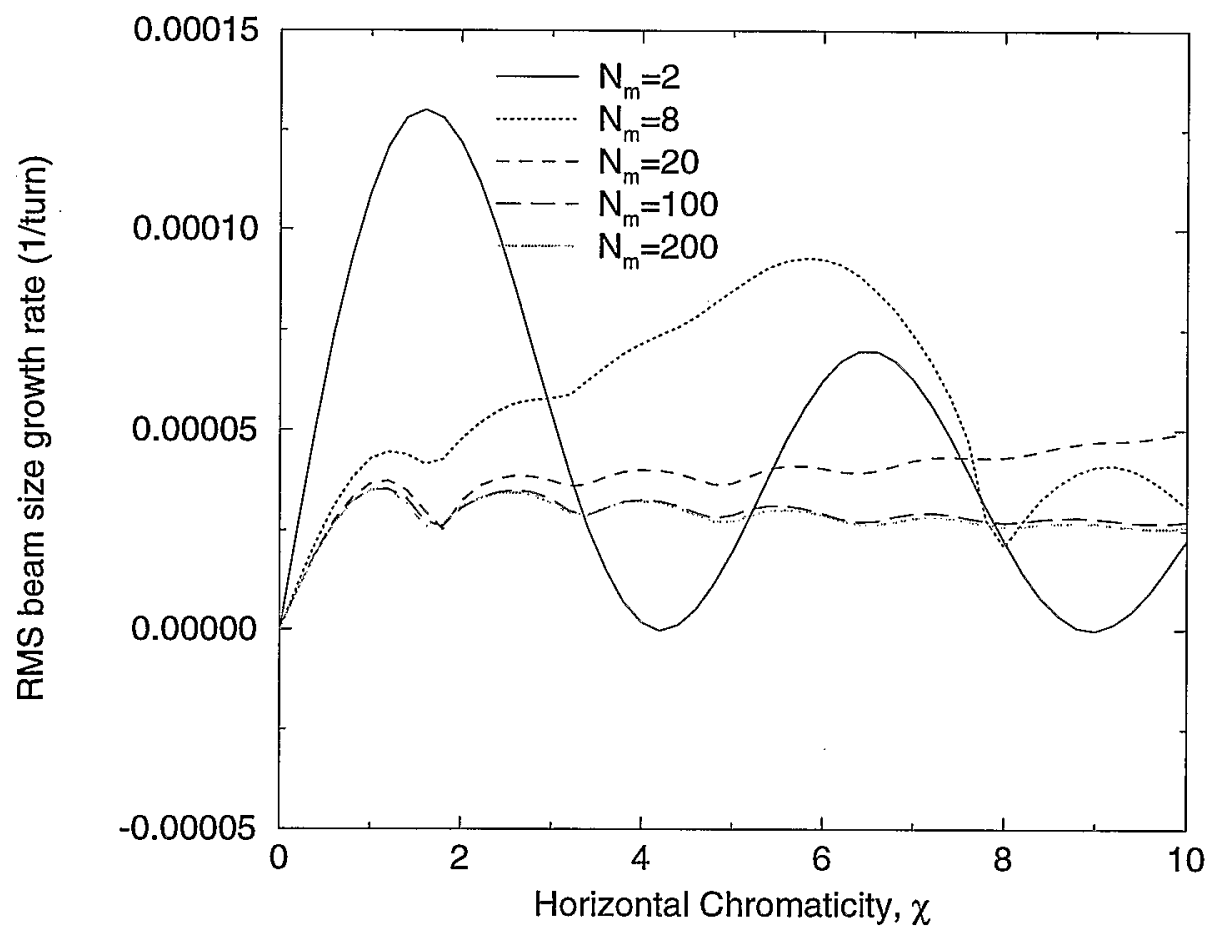

Figure 4: RMS beam size growth rate versus horizontal chromaticity for $N_{m}=$ $2,8,20,100,200$ macro-particles.

Fig. 4 shows simulation results for RMS beam size growth rate versus horizontal chromaticity for different numbers of macro-particles. The behavior for a few macro-particles changes a lot with the number of macro-particles, while for $N_{m} \gtrsim 100$ the behavior becomes independent of the number of macro-particles. Motion is apparently unstable for all chromaticities other than $\chi=0$. The growth rate is about 0.00003 turn $^{-1}$ and doesn't change much with chromaticity except for $\chi \lesssim 2.5$. The beam amplitude fluctuates wildly, and it's hard to get unambiguous growth rate measurements except over long time intervals. The trend of the curves in Fig.5 shows that finally they have the same growth rate as the RMS size shown in Fig.6. Initially the amplitude doesn't increase as fast as the RMS beam size, but in the long run it is also unstable. 


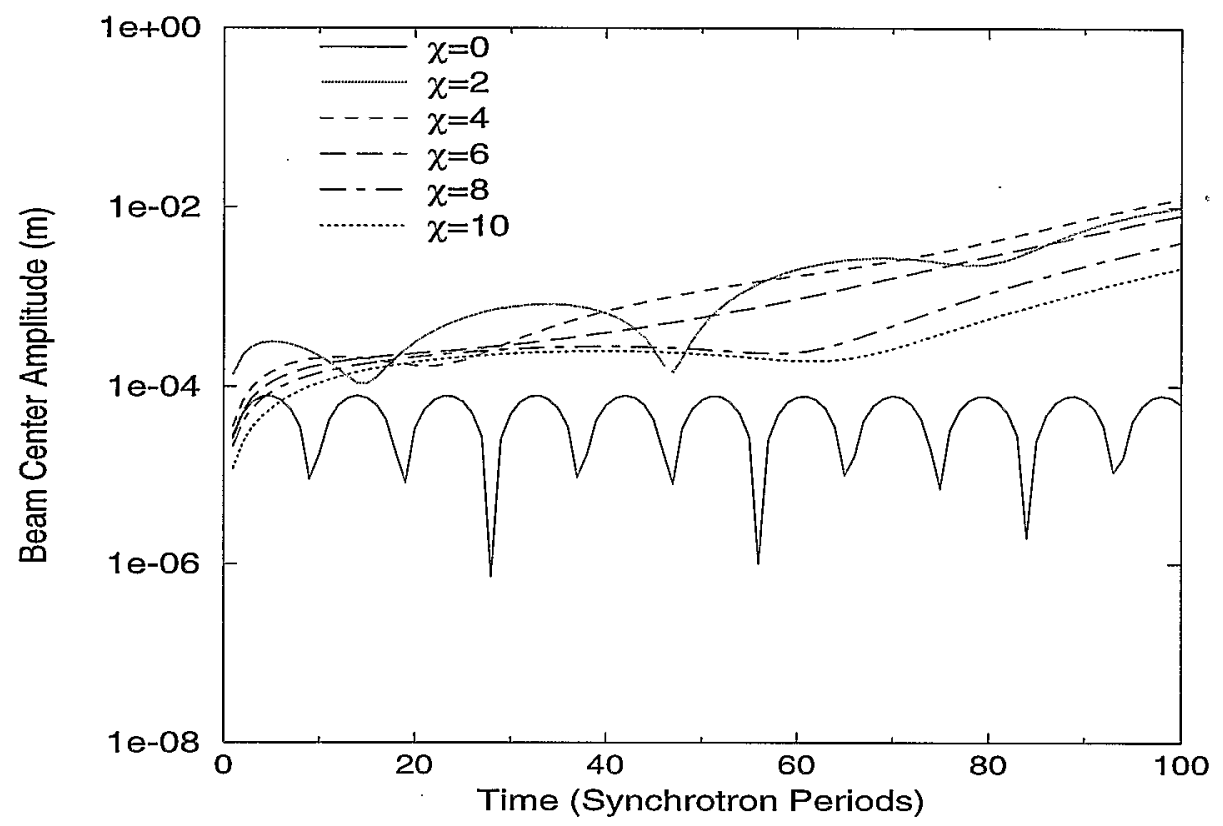

Figure 5: Beam amplitude versus time for $N_{m}=100$ macro-particles.

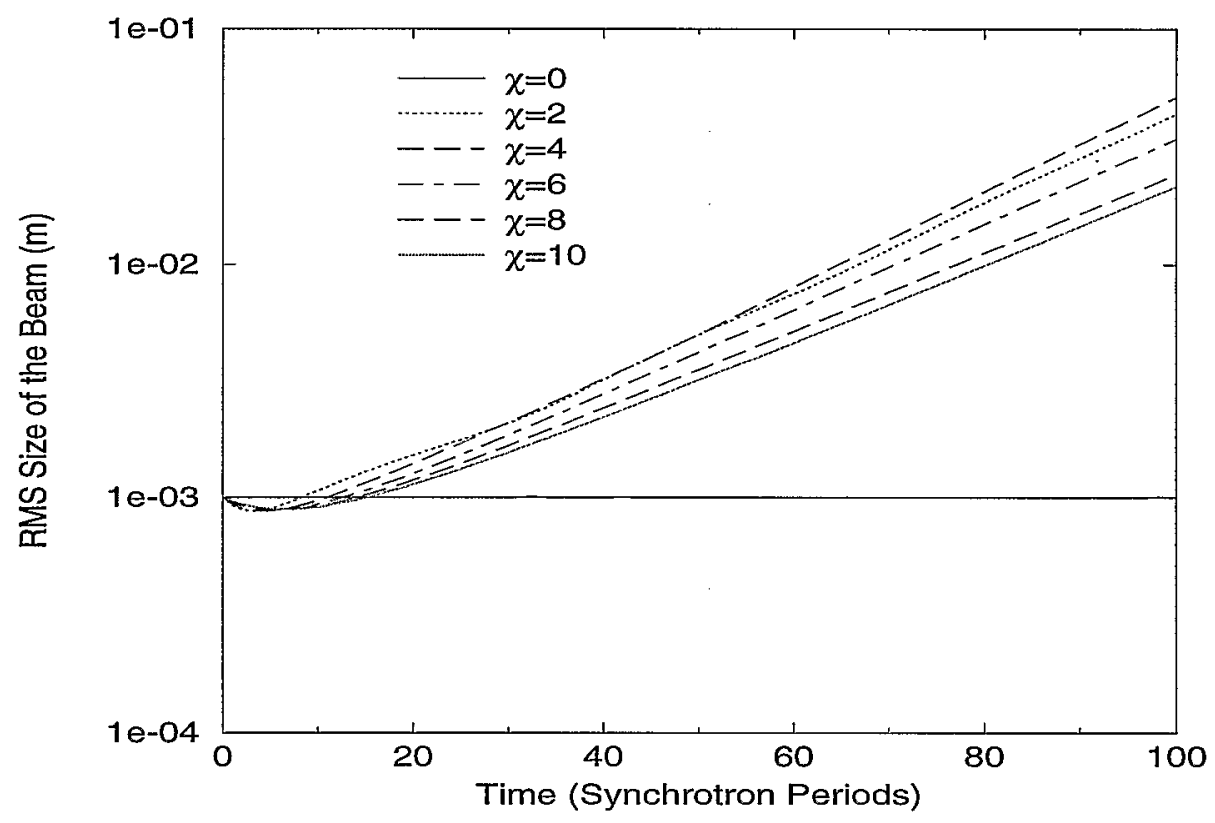

Figure 6: RMS beam size versus time for $N_{m}=100$ macro-particles. 
In the same spirit as in the analytical model, we know that there are $2 N_{m}$ eigenmodes in the transformation matrix representative of the action in each longitudinal oscillation period. But the mode with the strongest eigenvalue will dominate in the long run. And if any mode is unstable, it will be the source of head-tail instability for our physical observables.

We also tested for strong head-tail instability in KRAKEN with $N_{m}=100$ by setting the chromaticity to be zero. The results show that the criterion for strong head-tail instability is around $\operatorname{Re}(\Upsilon)=1.7$. This is reasonably close to the two macro-particle prediction that the beam should be unstable for $\operatorname{Re}(\Upsilon)>2$.

\section{Summary and conclusions}

From the simulation results by the generalization of the two macro-particle KRAKEN model, we conclude that the beam should be unstable due to the resistive wall head-tail instability if the chromaticity isn't zero. But we believe there are other self-stabilizing mechanisms such as Landau damping, and a more complete model should stabilize the beam for at least some chromaticities as in all accelerators.

We have used $N_{m}=100$ and 200 in the simulation by KRAKEN for tracking of 100 synchrotron periods. Besides distributing the macro-particles uniformly in longitudinal phase space by putting them on a circle, we also tried distributing them on a semi-circle, with essentially the same results. To further our simulation, we can try a finer distribution in longitudinal phase space, use a longer synchrotron oscillation period, and use more macro-particles. But in order to do these, the running time of KRAKEN will be much longer. To track $N_{m}=100$ macro-particles for 100 synchrotron periods on a SUN Ultra 1, with the chromaticity varying from 0 to 10 in intervals of 0.2 , the CPU running time is about 27 hours. The running time is roughly proportional to $N_{m}^{2}$.

\section{References}

[1] S. Peggs, V. Mane, "KRAKEN, a Numerical Model of RHIC Impedances", Dallas PAC, 1995, p. 3137.

[2] A. Chao, "Physics of Collective Beam Instabilities in High Energy Accelerators", New York: Jolnn Wiley and Sons, 1993.

[3] R. Kohaupt, DESY Report M-80/19, Hamburg, 1980.

[4] R. Talman, CERN Report ISR-TH/81-17, Geneva, 1981; R. Talman, Nucl. Instr. Meth., 193, 423, 1982.

[5] D. Edwards and M. Syphers, "An Introduction to the Physics of High Energy Accelerators", New York, John Wiley and Sons, 1993. 